\title{
Conditional expression of retrovirally delivered anti-MYCN shRNA as an in vitro model system to study neuronal differentiation in MYCN-amplified neuroblastoma
}

\author{
Jørn R Henriksen ${ }^{1,2}$, Bjørn Helge Haug ${ }^{1}$, Jochen Buechner ${ }^{1,2}$, Ellen Tømte ${ }^{1}$, Cecilie Løkke ${ }^{1}$, \\ Trond Flaegstad ${ }^{1,2}$, Christer Einvik ${ }^{1 *}$
}

\begin{abstract}
Background: Neuroblastoma is a childhood cancer derived from immature cells of the sympathetic nervous system. The disease is clinically heterogeneous, ranging from neuronal differentiated benign ganglioneuromas to aggressive metastatic tumours with poor prognosis. Amplification of the MYCN oncogene is a well established poor prognostic factor found in up to $40 \%$ of high risk neuroblastomas.

Using neuroblastoma cell lines to study neuronal differentiation in vitro is now well established. Several protocols, including exposure to various agents and growth factors, will differentiate neuroblastoma cell lines into neuron-like cells. These cells are characterized by a neuronal morphology with long extensively branched neurites and expression of several neurospecific markers.

Results: In this study we use retrovirally delivered inducible short-hairpin RNA (shRNA) modules to knock down MYCN expression in MYCN-amplified (MNA) neuroblastoma cell lines. By addition of the inducer doxycycline, we show that the Kelly and SK-N-BE(2) neuroblastoma cell lines efficiently differentiate into neuron-like cells with an extensive network of neurites. These cells are further characterized by increased expression of the neuronal differentiation markers NFL and GAP43. In addition, we show that induced expression of retrovirally delivered antiMYCN shRNA inhibits cell proliferation by increasing the fraction of MNA neuroblastoma cells in the G1 phase of the cell cycle and that the clonogenic growth potential of these cells was also dramatically reduced.
\end{abstract}

Conclusion: We have developed an efficient MYCN-knockdown in vitro model system to study neuronal differentiation in MNA neuroblastomas.

\section{Background}

Neuroblastoma is a childhood cancer arising from the sympaticoadrenal lineage of the neural crest. It is characterized by diverse clinical behaviours ranging from spontaneous regression, maturation to more benign forms (ganglioneuroblastoma or ganglioneuroma), to rapid tumour progression and death [1]. Amplification of the MYCN oncogene has been considered the most important prognostic factor for progressive disease and poor outcome. Despite intense efforts to elucidate a

\footnotetext{
* Correspondence: christer.einvik@uit.no

'Department of Pediatrics, University Hospital of North-Norway, 9038

Tromsø, Norway

Full list of author information is available at the end of the article
}

mechanism by which $M Y C N$ overexpression acts to promote the aggressive phenotype, the functional roles of the MYCN protein in neuroblastoma are poorly understood [2].

Several alternative mechanisms for neuroblastoma regression have been proposed over the past 20 years, although the principal mechanism underlying this peculiar phenomenon remains to be fully elucidated [3-5]. Tumour maturation via neuronal differentiation has recently been proposed as a plausible candidate mechanism to explain neuroblastoma regression [6]. Therefore, the study of neuroblastoma as a model system for the general process of tumour cell differentiation, as well as 
neuronal development, is important to reveal the secrets of both tumour maturation and spontaneous regression.

Using neuroblastoma cell lines to study neuronal differentiation in vitro is now well established. Induced neuron-like morphological and biochemical changes to the SH-SY-5Y neuroblastoma cell line was demonstrated almost 30 years ago using a bioactive phorbolester as the inducing agent [7]. Since then, a variety of various agents and growth factors have been shown to induce neuronal differentiation in many neuroblastoma cell lines (reviewed in [8]). Furthermore, specific suppression of $M Y C N$ expression using traditional antisense techniques or small interfering RNA molecules (siRNA) have also been shown to promote neuronal differentiation in several MYCN-amplified (MNA) neuroblastoma cell lines [9-12].

Plasmid and viral vector-based systems containing RNA polymerase III promoters for the expression of short hairpin RNAs (shRNAs) have become useful tools for modulating gene expression in mammalian cells [13]. Compared to siRNAs, the use of shRNAs to suppress gene function has been demonstrated to be more effective [14]. In addition, shRNA-based strategies offer the advantage of inducible expression in situations in which gene knockdown is expected to have a deleterious effect on the targeted cell.

Retroviral expression systems have proven to be useful tools for sustained long-term expression of transgenes in mammalian cells [15]. With the development of protocols to produce high-titer infectious, replicationincompetent retroviral particles, these expression systems are now commonly used for shRNA delivery.

In this study, we have developed a retroviral tetracycline-inducible anti-MYCN shRNA expression system to study MYCN knockdown-mediated neuronal differentiation in MNA neuroblastoma cell lines. We reveal that MNA neuroblastoma cell lines induced to express the anti-MYCN shRNAs efficiently undergo morphological and biochemical changes consistent with neuronal differentiation.

\section{Results and Discussion}

\section{Retrovirally delivered inducible shRNA expression in} HEK293T-Rex cells

We have previously developed and characterized an efficient doxycyclin (dox)-inducible variant of the $\mathrm{H} 1$ promoter (H1-2O2-US/DS) for shRNA expression in human cells. The H1-2O2-US/DS promoter was shown to be almost completely inactive in the non-induced state, while induction by dox yielded a high shRNA expression in transient transfection studies using an anti-luciferase (anti-luc) shRNA reporter system [16].

As a first step towards developing a long-term inducible shRNA expression system, we subcloned the previously described anti-luc shRNA constructs and the corresponding scrambled control shRNA into a retroviral expression vector. shRNA-expressing retroviruses were transduced into HEK293T-Rex cells followed by transient transfection of the luciferase reporter vector. Two days after transfection, cells were monitored for dox-regulated shRNA expression. Addition of dox to the transduced cells had only minor effects on luciferase expression as can be seen from both the inducible scrambled negative control (H1-2O2 Scr) and the H1-wt expressed anti-luc shRNA (H1-wt a-Luc) (Figure 1). Induction of anti-luc shRNA expression from the $\mathrm{H} 1-2 \mathrm{O} 2$ promoter by the addition of dox downregulated the luciferase reporter to levels similar to that observed for the H1-wt promoter. At the same time, no transcriptional activity was observed in the non-induced state (H1-2O2 aLuc, -dox) when compared to the scrambled shRNA control.

These data clearly show that retroviral delivery of the H1-2O2 US/DS-inducible shRNA expression system to HEK293T-Rex cells allows an efficient inducible expression of mature shRNA molecules.

\section{Efficient downregulation of $M Y C N$ expression in MNA neuroblastoma cell lines}

We have previously designed an anti-MYCN shRNA construct (aMN-887) for downregulation of $M Y C N$ oncogene expression in $M Y C N$-amplified (MNA) neuroblastoma cells [17]. This design procedure was based on the original Hannon protocol for shRNA construction, recommending $29 \mathrm{bp}$ stem structures [18]. We have

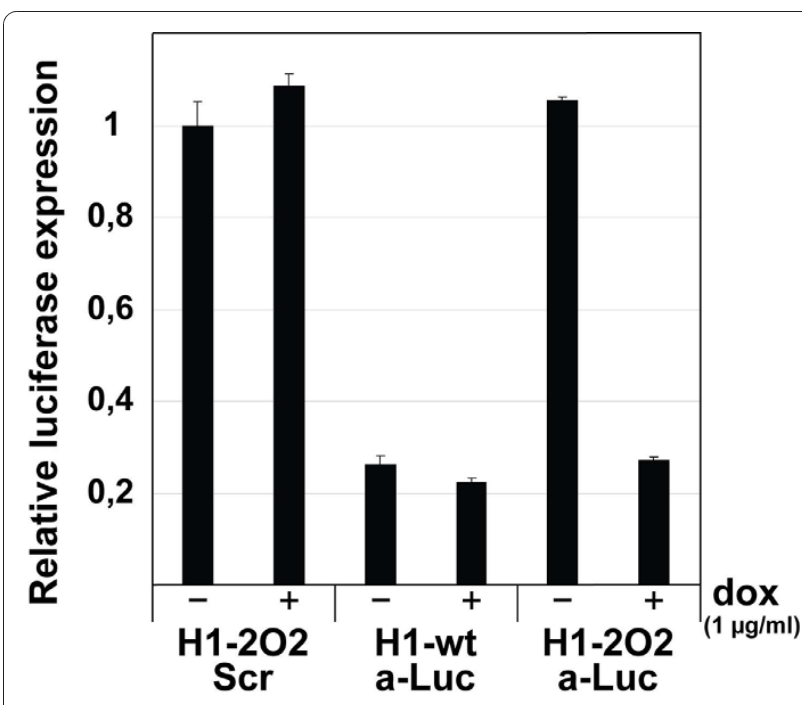

Figure 1 Retrovirally delivered inducible shRNA expression in HEK293 cells. TetR-expressing HEK293 cells (HEK293T-Rex) were transduced with retroviruses expressing anti-Luciferase (a-Luc) or scrambled (Scr) shRNAs. The a-Luc shRNA was expressed from a wt$\mathrm{H} 1$ or the inducible $\mathrm{H1}-2 \mathrm{O} 2$ promoter in the absence (-) or presence (+) of $1 \mu \mathrm{g} / \mathrm{ml}$ doxycyclin (dox) for $48 \mathrm{hrs}$. Error bars indicate SDs. 
now constructed a new anti- $M Y C N$ shRNA construct (aMN-1658) targeting the 3'UTR of the MYCN mRNA.

The MNA Kelly and SK-N-BE(2) neuroblastoma cell lines were transiently transfected with plasmids expressing the aMN-887 or aMN-1658 shRNAs. A scrambled shRNA ( $\mathrm{Scr}$ ) construct was used as the control. Three days after transfection, cells receiving the aMN (anti$M Y C N)$ shRNAs appeared to have a more neuron-like phenotype characterized by neurite outgrowth, while cells transfected with the control shRNA did not reveal any morphological alterations as compared to nontransfected cells (Figure 2). Western immunoblot and quantitative real-time RT-PCR analysis confirmed specific suppression of the MYCN protein and $M Y C N$ mRNA in both cell lines, respectively (Figure $3 a$ and Figure $3 b)$.

Consistent with previous data, an inverse correlation between $M Y C N$ and $c-M Y C$ mRNA expression was also confirmed (Figure 3c) [19,20]. The observed morphological changes were documented biochemically by increased expression of the early neuronal differentiation marker NFL (Figure 3d). Furthermore, expression of aMN-1658, but not of aMN-887, resulted in a marked increase in the late neuronal differentiation marker GAP43, which is known to be involved in axonal outgrowth and synapse formation (Figure 3e) [21]. This observation is consistent with the more extensive neuronal differentiation observed for both Kelly and SK-N-BE (2) cells transfected with aMN-1658 compared to cells transfected with aMN-887 (Figure 2).

These data show that aMN-1658 is an efficient anti$M Y C N$ shRNA construct which suppresses $M Y C N$ expression and induces prominent neuronal differentiation in MNA neuroblastoma cell lines. For that reason, the aMN-1658 shRNA was used in the following research to knock down MYCN expression.

Previous reports document conflicting results on cell cycle distribution data of SK-N-BE(2) cells treated with anti-MYCN siRNAs. While Yu et al. reported no apparent difference in the fraction of G1 cells after siRNA treatment, Bell et al. showed that $M Y C N$ siRNA treatment increased the $\mathrm{G} 1$ population by $8.1 \%$ compared to

\section{A}

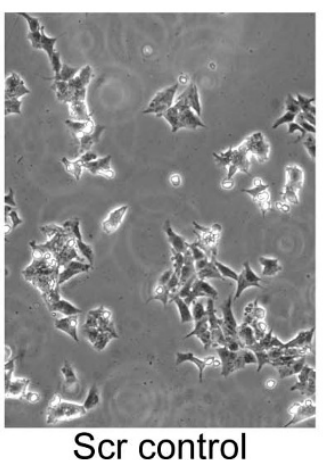

B

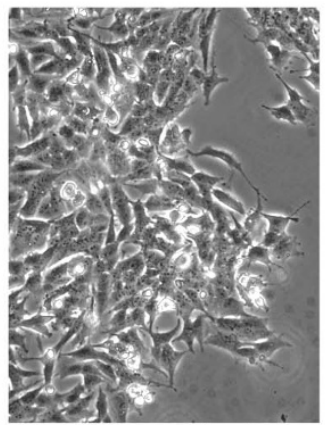

Scr control

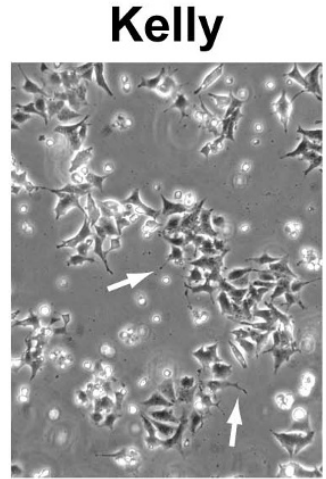

aMN-887
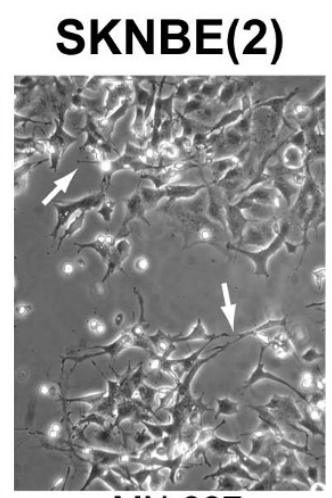

aMN-887

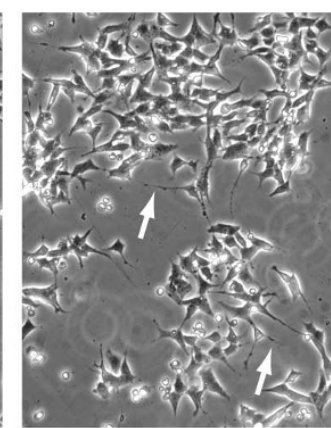

$\mathrm{aMN}-1658$

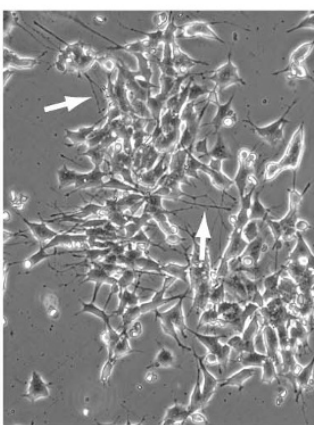

$\mathrm{aMN}-1658$

Figure 2 MYCN knockdown induces neurite outgrowth in MNA neuroblastoma cell lines. MNA Kelly (A) and SK-N-BE(2) (B) cells transiently transfected for 3 days with anti-MYCN (aMN-887 and aMN-1658) and scrambled (Scr control) shRNA expressing plasmids. Cells expressing the aMN shRNAs show neurite outgrowth characteristic of neuronal differentiated cells. White arrows indicate neurite outgrowth. 


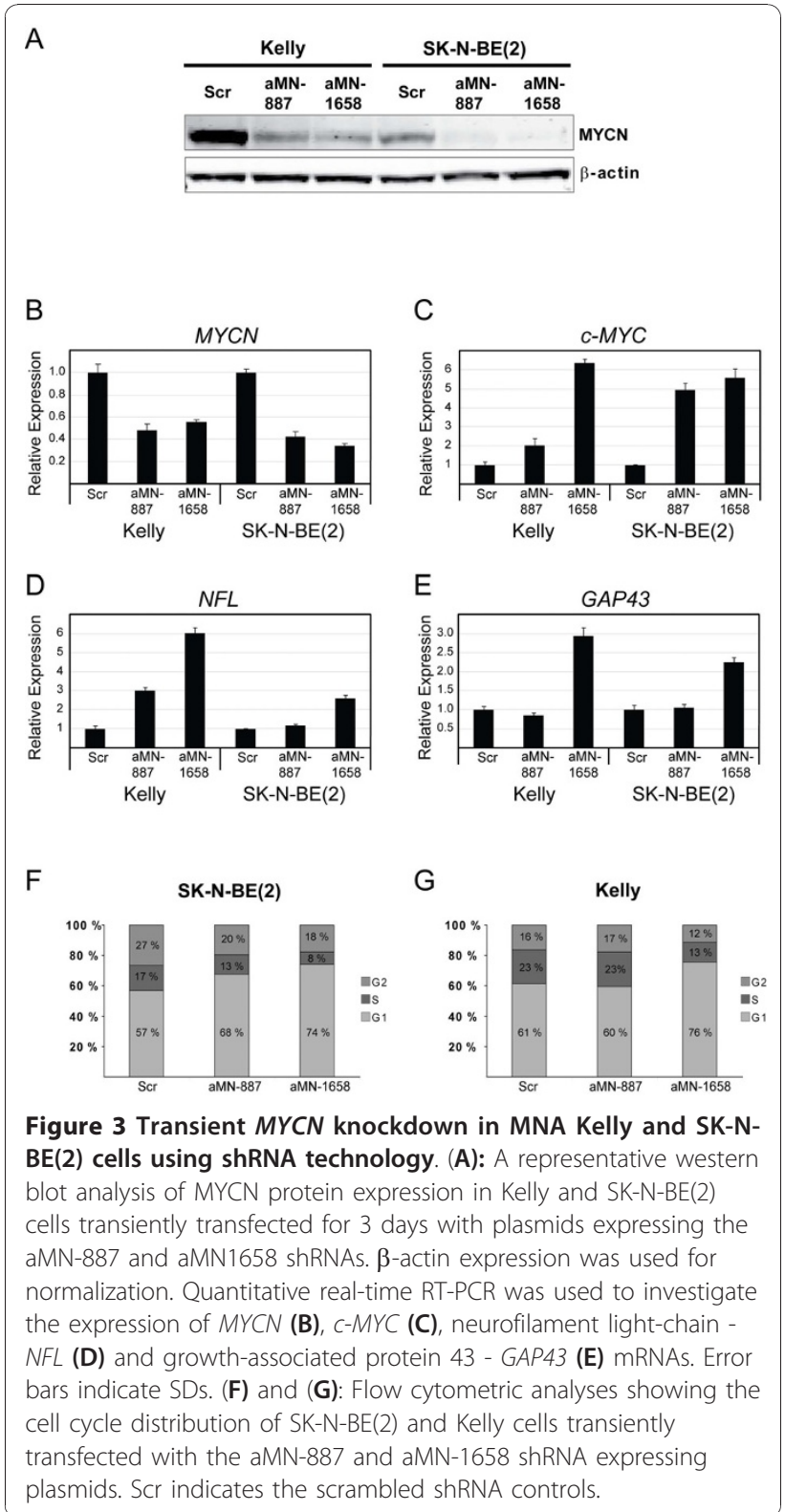

a negative scrambled control siRNA [9,22]. In order to investigate the effect of shRNA-mediated MYCN knockdown on the cell cycle distribution pattern, we transiently transfected SK-N-BE(2) cells with plasmids expressing the aMN-887, aMN-1658 or a scrambled shRNA from a wt $\mathrm{H} 1$ promoter. Three days posttransfection the cell cycle distribution pattern was monitored using flow cytometry. The fraction of cells in the G1 phase of the cell cycle increased from $57 \%$ (Scr) to $74 \%$ for SK-N-BE(2) cells transfected with the aMN-1658 shRNA (Figure 3f). Similar results were obtained with aMN1658-transfected Kelly cells (Figure 3g).

A discrepancy between the knockdown levels for the MYCN protein (85-94\%) and mRNA (45-70\%) was observed in our MYCN shRNA knockdown experiments, with similar results being previously reported for $M Y C N$ siRNA knockdown by others [23]. In order to further document that only the expression of the MYCN shRNAs results in reduced MYCN expression, we set up additional experiments. The complete MYCN 3'UTR sequence, including the aMN-1658 target sequence, was cloned behind a luciferase reporter gene in the PMIR-REPORT vector (Ambion). Co-transfecting this reporter vector and the aMN-shRNA expressing plasmids into HEK-293 cells demonstrated the specific knockdown, monitored as an $80 \%$ reduction in luciferase activity, by the aMN-1658 shRNA (Additional file 1a). When aMN-1658 was cotransfected with a control reporter vector containing a 3'UTR lacking the aMN-1658 target sequence (Luc NT3'UTR), no change in luciferase expression was observed, and similar results were obtained in the non-MNA neuroblastoma cell line SH-SY-5Y (data not shown).

In addition, we co-expressed the aMN-shRNA constructs and the $M Y C N$ cDNA lacking the 3'UTR sequence in a MNA neuroblastoma cell line. Downregulation of both the endo- and exogenously expressed MYCN protein was only observed for the aMN-887 shRNA targeting the coding region of $M Y C N$. The aMN-1658 shRNA was not able to suppress the exogenously expressed MYCN cDNA lacking the 3'UTR structure (Additional file 1b).

These experiments show that the aMN-1658 shRNA specifically reduces expression of mRNAs containing the MYCN 3'UTR target. However, we have not been able to confirm or rule out the possibility that a part of the MYCN protein reduction by aMN-1658 shRNA knockdown is mediated by a miRNA-like mechanism not involving mRNA degradation. Nonspecific cellular effects induced by shRNA expression were excluded by showing that the expression level of several genes involved in the interferon response (OAS2, MX1, IFITM 1 and ISGF3 $\gamma$ ) remained unaltered during transfection of various shRNA constructs (data not shown).

\section{Retrovirally delivered inducible anti-MYCN shRNA expression in MNA neuroblastoma cell lines}

In order to generate a long-term inducible anti-MYCN shRNA expression system, we first cloned the aMN1658 shRNA module behind the inducible H1-2O2-US/ DS promoter and then gated the resulting construct into a retroviral expression vector to generate $\mathrm{pRV}$ 1658. A similar vector expressing a scrambled shRNA was constructed as a negative control (pRV-Scr).

Kelly and SK-N-BE(2) neuroblastoma cell lines stably expressing the TetR (tetracycline repressor) were transduced with the retroviruses RV-1658 and RV-Scr and then incubated for 6 days in a growth medium both with and without dox. As can be seen from Figure $4 \mathrm{a}$ 


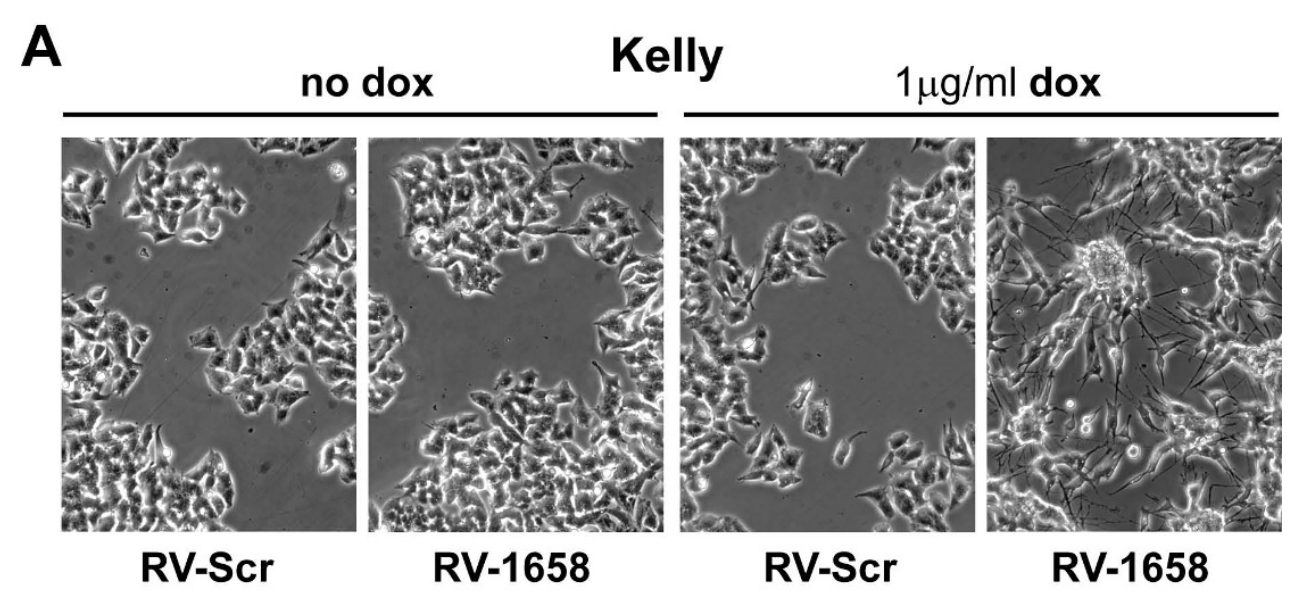

B

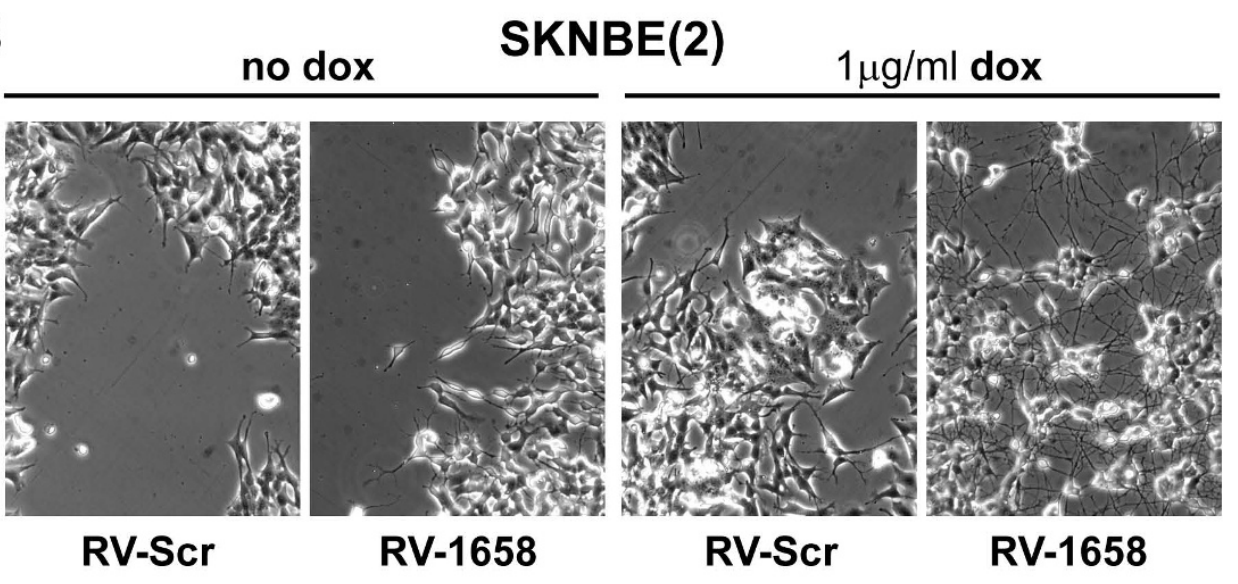

C

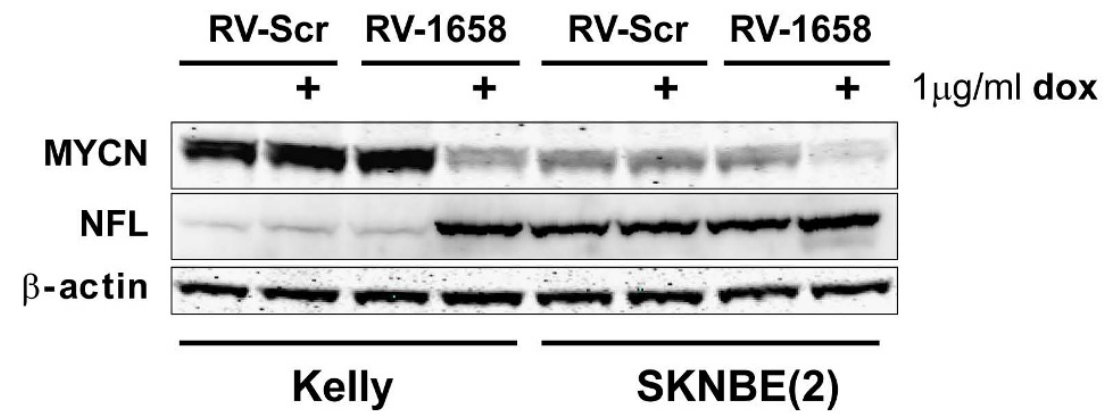

Figure 4 Inducible $M Y C N$ knockdown in retrovirally transduced MNA neuroblastoma cell lines. Representative micrographs showing the morphology of Kelly (A) and SK-N-BE(2) (B) cells transduced with retroviruses delivering the dox-inducible aMN-1658 shRNA module (RV-1658). RV-Scr indicates the cells receiving retroviruses delivering an inducible scrambled shRNA module. (C): A representative western blot showing MYCN and NFL expression in Kelly and SK-N-BE(2) cells transduced with retrovirus RV-1658 and induced to express the shRNA by addition of $1 \mu \mathrm{g} / \mathrm{ml}$ doxycyclin (dox). 
and Figure 4b, only the cells induced to express the aMN-1658 shRNA demonstrated prominent neuronal differentiation. The lack of morphological changes in non-induced RV-1658 transduced cells indicates that the $\mathrm{H} 1-2 \mathrm{O} 2$ promoter shows minimal transcriptional leakage in the absence of dox. The addition of dox to RV-Scr transduced cells had no significant effect on the cell morphology. Western blot analysis of the retrovirally transduced Kelly and SK-N-BE(2) cells revealed efficient repression of $\mathrm{MYCN}$ expression in cells induced to express the aMN-1658 shRNA (Figure 4c). Similar to the observations made in transient expression analyses, the MYCN mRNA levels were reduced to a lesser extent when compared to the western blot data (data not shown and Figure 5b).

\begin{tabular}{|c|c|c|c|c|c|c|c|c|c|c|c|}
\hline \multirow{3}{*}{ A } & \multicolumn{5}{|c|}{ Kelly } & \multicolumn{5}{|c|}{ SKNBE(2) } & \multirow[b]{2}{*}{ time (days) } \\
\hline & 0 & 3 & 6 & $3 / 3$ & 6 & 0 & 3 & 6 & $3 / 3$ & 6 & \\
\hline & - & + & + & $+1-$ & - & - & + & + & $+1-$ & - & $1 \mu \mathrm{g} / \mathrm{ml}$ dox \\
\hline MYCN & $=$ & - & $t=$ & - & $=$ & $=$ & $=$ & 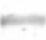 & - & - & \\
\hline GAP43 & $=$ & $=$ & - & $=$ & $=$ & $=$ & & 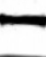 & & - & \\
\hline
\end{tabular}

B

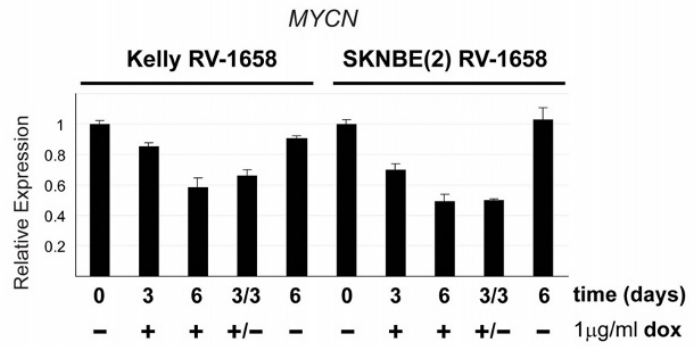

C

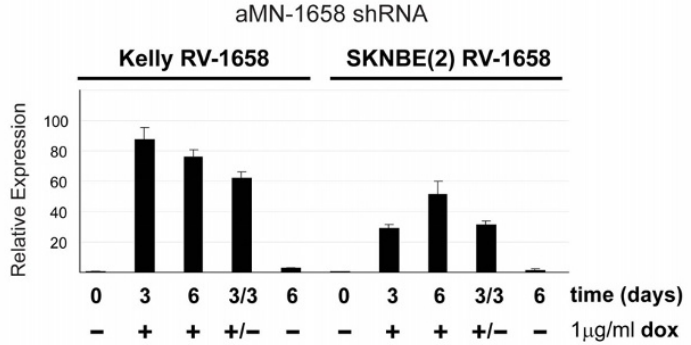

Figure 5 Induced anti-MYCN shRNA expression decreases MYCN and increases GAP43 expression in MNA neuroblastoma cells. (A) A representative western blot analysis of MYCN, GAP43 and $\beta$-actin protein expression in Kelly and SK-N-BE(2) cells induced to express the aMN-1658 shRNA. Real-time RT-PCR analysis of MYCN mRNA (B) and aMN-1658 shRNA (C) expression was performed on total RNA isolated from Kelly and SK-N-BE(2) cells treated as described. Cells were transduced with retrovirus RV-1658 and incubated for the indicated numbers of days in the presence $(+)$ or absence (-) of $1 \mu \mathrm{g} / \mathrm{ml}$ doxycyclin (dox). $3 / 3$ and $+/$ - indicate that the cells were incubated for 3 days in the presence of dox, followed by 3 days in the absence of dox. Error bars indicate SDs.
In order to investigate the reversibility of the inducible retroviral shRNA expression system, we performed a time-course experiment in which the aMN-1658 expression was turned on for 3 days before removing dox from the media for another 3 days to eliminate shRNA expression.

Compared to the differentiated cells continuously exposed to aMN-1658 shRNA for 6 days, no significant change in cell morphology was observed when shRNA expression was turned off (data not shown). When the MYCN protein and mRNA levels were measured during the 6 days of shRNA induction, a steady decrease in MYCN expression was observed. Removal of dox from the media after 3 days of exposure did not efficiently recover MYCN expression. Consistent with the observed neuron-like phenotype, GAP43 protein levels remained high after dox removal (Figure $5 \mathrm{a}$ and Figure 5b).

By the use of a quantitative RT-PCR protocol designed to measure the mature antisense shRNA strand, we observed a maximum 90-fold increase in aMN-1658 RNA after 3 days of dox induction in the Kelly cells (Figure 5c). After additional 3 days of induction, a slight decrease in shRNA expression was observed. For the SK-N-BE(2) cell line, a maximum 50-fold increase in shRNA expression was observed after 6 days of continuous induction. Removing the inducing agent from the media did not efficiently cease shRNA expression. We suggest that this lack of reversibility is most likely due to high intracellular shRNA stability and/or insufficient removal of dox from the media.

We also analysed the expression of the neuronal differentiation markers NFL and GAP43 in Kelly and SK$\mathrm{N}-\mathrm{BE}(2)$ cells transduced with shRNA-expressing retroviruses. Consistent with the observed morphological changes towards a neuronal phenotype, both differentiation markers increased upon aMN-1658 induction (Figure 5a, Additional file $2 \mathrm{a}$ and Additional file $2 \mathrm{~b}$ ). Quantitative real-time PCR revealed a 5-8 fold increase in NFL mRNA and a 3-fold increase in GAP43 mRNA in both cell lines induced to express the aMN-1658 shRNA.

SK-N-BE(2) has previously been described as a noradrenergic neuroblastoma cell line based on expression of enzymes involved in neurotransmitter synthesis [24]. In order to get further information about the neuronal phenotype observed in our experiments, we investigated the expression levels of the cholinergic markers choline acetyl transferase (ChAT) and vesicular acetylcholine transporter $(V A C h T)$, and the catecholaminergic marker tyrosine hydroxylase $(T H)$. When Kelly and SK-N-BE(2) cells were induced to differentiate by $M Y C N$ knockdown we observed increased mRNA expression levels of ChAT and $V A C h T$. The mRNA levels of $T H$ decreased during the same treatment (Additional file 2c). These data are 
consistent with a switch towards a more sympathetic cholinergic neuronal phenotype. A similar switch in neuronal phenotype, including increased NPY expression, has previously been reported for a neuroblastoma cell line induced to differentiate by a combination of retinoic acid (RA) and brain-derived neurotropic factor (BDNF) [25]. Differentiated neuroblastoma tumours have recently been shown to have cholinergic characteristics as compared to poorly-differentiated or undifferentiated neuroblastomas [26].

In summary, our data show that retroviral delivery of inducible anti-MYCN shRNAs to MNA neuroblastoma cells efficiently reduces MYCN protein expression and induces neuronal differentiation.

\section{Induced anti-MYCN shRNA expression inhibits cell proliferation and clonogenic growth of MNA neuroblastoma cells}

The Alamar Blue Assay was used to measure cell proliferation in MNA neuroblastoma cells induced to express the anti-MYCN shRNA from retroviral vectors. Figure 6 shows that the rate of cell proliferation was selectively

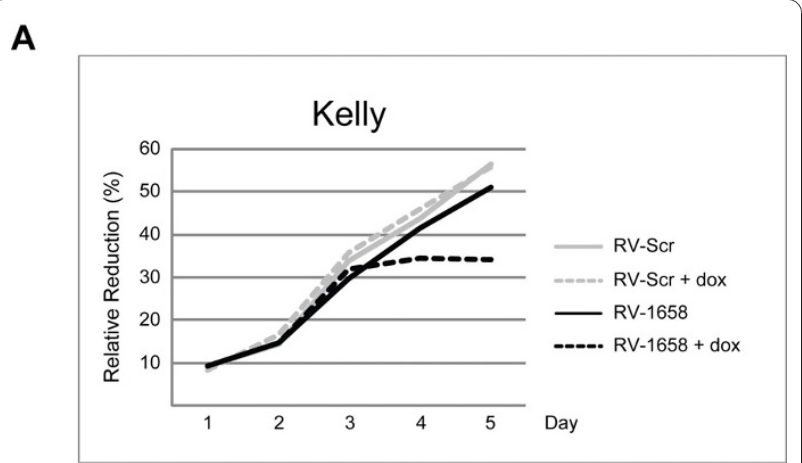

B

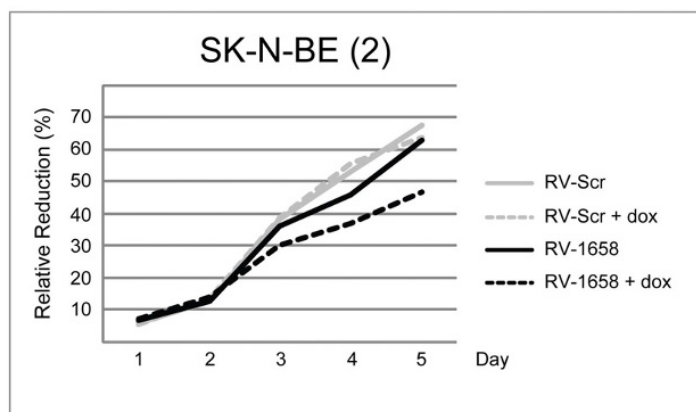

Figure 6 The proliferative inhibition effect of induced MYCN knockdown in MNA neuroblastoma cells. The Alamar Blue Assay was used to measure cell viability in Kelly (A) and SK-N-BE(2) (B) cells transduced with retrovirus RV-Scr (scrambled control) and RV1658 (anti-MYCN). The addition of doxycyclin (+ dox) induces expression of the shRNAs. $\mathrm{AB}$ reduction = Alamar Blue reduction. decreased from day 2 in SK-N-BE(2) and day 3 in Kelly cells induced to express the aMN-1658 shRNA. Cells expressing the scrambled shRNA showed no differences in cell proliferation due to the presence or absence of dox. The slight reduction in cell proliferation observed in the non-induced aMN-1658 transduced cells is most likely due to a small leakage from the shRNA-expressing promoter. The delayed decrease in cell proliferation observed in Kelly cells is consistent with the $\sim 5$-fold higher MYCN mRNA levels in comparison to MNA SK$\mathrm{N}-\mathrm{BE}(2)[27,28]$.

The observed reduction in the proliferation of cells induced to express the anti-MYCN shRNA was further investigated by flow cytometry in order to elucidate the cell cycle distribution pattern. Induced $M Y C N$ knockdown in the MNA Kelly cells increased the fraction of G1 cells from $63 \%$ to $72 \%$. For the MNA SK-N-BE(2) cells the fraction of G1 cells increased from $46 \%$ to $57 \%$ upon induction of aMN-1658 expression. Additon of dox alone had no effect on the cell cycle distribution pattern in cells transduced with retroviruses expressing a scrambled shRNA control (Additional file 3).

Finally, in vitro clonogenic assays were used to measure the reproductive cell survival in MNA neuroblastoma cells induced to differentiate by $M Y C N$ knockdown. Figure 7 shows that $M Y C N$ knockdown leads to dramatic growth inhibition only in MNA neuroblastoma cells induced to express the anti-MYCN shRNA.

Together, these data indicate that the induced expression of retrovirally delivered anti-MYCN shRNA inhibits cell proliferation by increasing the fraction of MNA neuroblastoma cells in the G1 phase of the cell cycle. The clonogenic growth potential of these cells was also dramatically reduced.

\section{Conclusion}

We have developed an efficient $M Y C N$-knockdown in vitro model system to study neuronal differentiation in MNA neuroblastomas.

\section{Methods}

Cell cultures and transfection

The human $M Y C N$-amplified neuroblastoma cell lines Kelly and SK-N-BE(2), and their derivatives SKNBE(2)TetR and Kelly-TetR (constitutively expressing the Tetracycline Repressor), were cultivated as previously described [16]. HEK293 and Hek293 Phoenix-amphopack (a kind gift from Dr. James Lorens) cells were grown in a Dulbecco's modified Eagle's medium (DMEM) [29]. HEK293-TREx cells (Invitrogen, Carlsbad, CA, USA) were grown in DMEM with $15 \mu \mathrm{g} / \mathrm{ml}$ blasticidin. All media were supplemented with 10\% FBS. Cells were maintained in a humidified $37^{\circ} \mathrm{C}$ incubator 


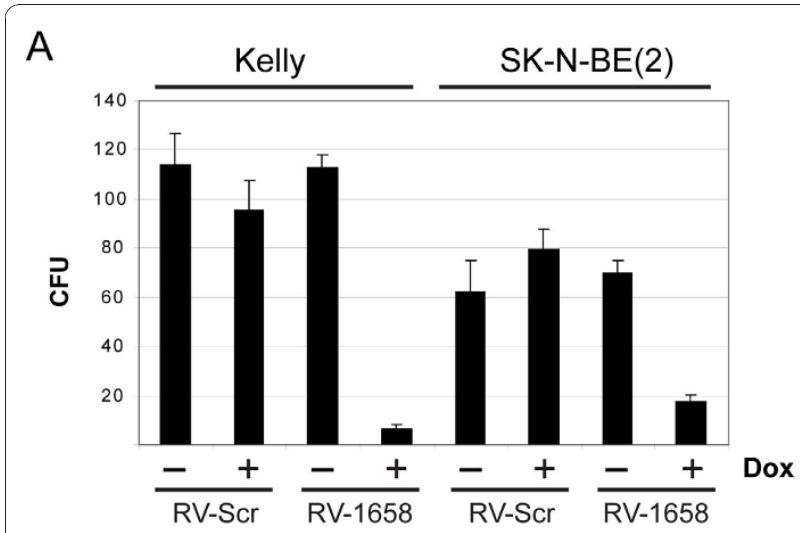

B

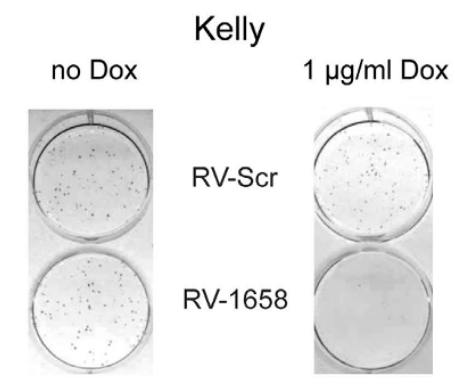

Figure 7 Reproductive cell survival in MNA neuroblastoma cells after MYCN knockdown. (A): Graphic presentation of colony forming units (CFU) after induced expression (+ dox) of scrambled (Scr) and anti-MYCN (aMN1658) shRNA in Kelly and SK-N-BE(2) cells. (B): Representative pictures of CFU from retrovirally transduced Kelly cells as presented in A.

with $5 \% \mathrm{CO}_{2}$, supplied with a fresh complete medium every 3 days and subcultured before confluence was reached.

Cell lines were transfected using Lipofectamine 2000 (Invitrogene) according to the manufacturer's instructions.

\section{Molecular cloning}

The number denotation of anti-MYCN (aMN) shRNAs describes the first position of the shRNA target recognition site in the MYCN cDNA sequence (NM_005378).

Construction of the following shRNAs was performed by annealing a sense and an antisense oligonucleotide: aMN-887 (ON106/ON107) and aMN-1658 (ON413/ $\mathrm{ON} 414)$. Oligonucleotide $(\mathrm{ON})$ sequences are listed in Additional file 4. Annealed oligonucleotides were ligated into BglII/HindIII digested pENTR-H1-wt or pENTRH1-2O2-US/DS vectors as previously described [16] to generate paMN-887/H1wt, paMN-887/H1-2O2, paMN1658/H1wt and paMN-1658/H1-2O2. Scrambled (Scr)
shRNA expressing vectors $\mathrm{pScr} / \mathrm{H} 1 \mathrm{wt}$ and $\mathrm{pScr} / \mathrm{H} 1-2 \mathrm{O} 2$ were made in a similar way using ON110/ON111. The vectors paMN-887/H1wt and paMN-1658/H1wt were used in transient transfection studies.

To generate the inducible retroviral expression vectors pRV-1658 and pRV-Scr, the shRNA expression modules from paMN-1658/H1-2O2 and $\mathrm{pScr} / \mathrm{H} 1-2 \mathrm{O} 2$ respectively, were gated into the retroviral destination vector L193 RRI-GreenattR1ccdBCmRattR2 (a kind gift from Dr. David Micklem) using the Gateway LR Clonase Enzyme Mix (Invitrogen). L193 RRI-GreenattR1ccdBCmRattR2 is based on the L071 RRI-Green vector [30]. The U6promoter/shRNA cloning cassette of L071 (flanked by HindIII and SalI sites) was replaced with an attR1-ccdB-CmR-attR2 Gateway cloning cassette derived from pDONR221 (Invitrogen). This cassette allows L193 to be used as a Gateway Destination Vector and requires that the vector is propagated in a ccdB-resistant strain. All plasmid constructs were verified by DNA sequencing.

\section{Production of retroviruses, transduction and induction of} shRNA expression

Retroviral destination vectors pRV-1658 and pRV-Scr were transfected into the Hek293-Phoenix packaging cell line seeded in 6-well plates. $24 \mathrm{~h}$ after transfection, the culture media were replaced by fresh media corresponding to the cell line being transduced. After another $24 \mathrm{~h}$ of incubation, the media were passed through a Millex HV $0.45 \mu \mathrm{m}$ PVDF filter (Millipore, Bedford, MA, USA) to isolate retroviral particles. Polybrene (Sigma, St. Louis, MO, USA) was added to a final concentration of $4 \mu \mathrm{g} / \mathrm{ml}$.

Cells were transduced by replacing the growth media with the solution of isolated retroviruses containing polybrene. The following day the virus-containing media were replaced with normal growth media containing puromycin (HEK293T-Rex and Kelly: $200 \mathrm{ng} / \mathrm{ml}$, SK-N$\mathrm{BE}(2): 2500 \mathrm{ng} / \mathrm{ml})$.

Induction of shRNA expression was performed by the addition of $1 \mu \mathrm{g} / \mathrm{ml}$ doxycyclin (dox) to the media.

\section{Quantitative real-time RT-PCR}

Total RNA was extracted using the Qiagen miRNeasy Mini Kit (Qiagen, Hilden, Germany). $1 \mu \mathrm{g}$ total RNA was reverse transcribed in $20 \mu \mathrm{l}$ using a Qiagen miScript Reverse Transcription Kit according to the manufacturer's instructions. Real-time PCR analysis was performed in $25 \mu \mathrm{l}$ reactions using Power SYBR Green PCR Master Mix (Applied Biosystems, Warrington, $\mathrm{UK})$ as recommended by the manufacturer $(2.5 \mu \mathrm{l}$ of a $20 x$-diluted RT-reaction as template, $0.2 \mu \mathrm{M}$ of each 
primer). UBC (ON56/ON57) and PPIA (ON174/ ON175) were used as reference genes for normalizing expression levels of $M Y C N$ (ON440/ON441 and ON145/146 were used for comparing the effect of aMN-887 and aMN-1658), NFL (ON58/ON59), GAP43 (ON298/ON299), c-myc (ON100/ON101), TH (ON453/ ON454), ChAT (ON516/ON517), VAChT (ON518/ ON519) and NPY (ON060/ON061). Primer sequences are listed in Additional file 4.

Quantification of the aMN-1658 shRNA was performed using miScript SYBR green PCR Kit (Qiagen) in $50 \mu \mathrm{l}$ reactions with $1 \mu \mathrm{l}$ of the $20 \times$-diluted $\mathrm{RT}$ reaction as template. Specific primers for quantifying aMN-1658 shRNA were ordered as a custom miScript Primer Assay from Qiagen using 5'CACACAAGGUGACUUCAACAGUU3' as the mature miRNA sequence, and were used at concentrations specified by the manufacturer. $U B C$ and PPIA were used for normalizing. All quantitative PCR reactions were performed using the Applied Biosystems 7300 Real-Time PCR System (Applied Biosystems) with thermal profiles as recommended by the manufacturer. The fold change in mRNA and miRNA levels were calculated using the $\Delta \Delta C T$ method with the qBASE software [31].

\section{Western blotting}

Cells were trypsinized and lysed in a Tropix Lysis Solution (Bedford, MA, USA). Lysate cleared by centrifugation was measured for total protein using Bio-Rad $D C$ protein assay (Bio-Rad, Hercules, CA, USA), and $35 \mu \mathrm{g}$ protein were loaded in each well on pre-casted NuPAGE 4-12\% Bis-Tris Gels (Invitrogen). The separated proteins were transferred to an Immobilon-FL PVDF transfer membrane (Millipore) and blocked for 1 hour at room temperature in Odyssey Blocking Buffer (LI-COR, Lincoln, NE, USA) before incubation at $4^{\circ} \mathrm{C}$ overnight with the following primary antibodies: mouse antiNMYC (Calbiochem/Merck, Darmstadt, Germany), mouse anti-GAP43 (Abcam, Cambridge, UK), goat anti-NFL (Santa Cruz Biotech., Santa Cruz, CA, USA) and rabbit anti- $\beta$ Actin (Sigma) diluted in the blocking buffer. Secondary antibodies were goat anti-rabbit IRDye $800 \mathrm{CW}$ (Rockland, Gilbertsville, PA, USA) and goat anti-mouse Alexa Fluor 680 (Invitrogen). Antibody binding was detected using the Odyssey Infrared Imaging System (LI-COR). NFL was detected by HRP conjugated rabbit anti-goat secondary antibody (DAKO, Glostrup, Denmark) and SuperSignal West Pico Chemiluminescent Substrate (Pierce, Rockford, IL, USA).

\section{Luciferase assay}

Retrovirus-transduced HEK293T-Rex cells were seeded in 12-well plates. $48 \mathrm{hrs}$ following induction with $1 \mu \mathrm{g} /$ ml dox, each well was transfected with $0.02 \mu \mathrm{g}$ pGL4.75 [hRluc/CMV] Vector (Promega, Madison, WI, USA) and $0.1 \mu \mathrm{g}$ pGL3 Control Vector (Promega). Firefly and renilla luciferace activity were measured after 48 hrs using the Dual-Luciferase Reporter Assay System (Promega) according to the manufacturer's instructions. Luminescence was measured on a Luminoskan Ascent luminometer (Thermo Sci., Waltham, MA, USA). Renilla luciferase was used to normalize the data, and all experiments were performed in triplicate.

\section{Clonogenic assay}

The cells were grown for 7-11 days with replacement of the media every third day. On the last day of the experiment, the cells were washed once in $1 \times \mathrm{PBS}$ and stained with the clonogenic reagent (50\% EtOH, 0.25\% 1,9-dimethyl-methylene blue) for $45 \mathrm{~min}$. Cells were washed twice in PBS before visible colonies were counted.

\section{Cell viability assay}

The cytotoxic effect of induced MYCN knockdown on MNA neuroblastoma cells was analysed using the Alamar Blue Assay according to the manufacturer's instructions. In brief, the cells were seeded at a density of 40000 (Kelly) and 15000 (SK-N-BE(2)) cells per well in 12 -well plates. Media were replaced every second day. On the indicated days, $100 \mu \mathrm{l}$ of Alamar Blue solution were added to each well and incubated for $4 \mathrm{hrs}$ at $37^{\circ} \mathrm{C}$. Absorbance at 570 and $600 \mathrm{~nm}$ was measured on a plate reader and the relative reduction of Alamar Blue was calculated as described by the manufacturer. The calculated average relative reduction from three independent experiments was calculated.

\section{Flow cytometric analysis of cell cycle distribution}

Cells were harvested using Trypsin-EDTA (SigmaAldrich) and washed once in $1 \times$ PBS. The cells were then fixed for $2 \mathrm{hrs}$ in ice-cold $70 \% \mathrm{EtOH}$. After fixation, the $\mathrm{EtOH}$ was removed by centrifugation and the cells were washed once in $1 \times$ PBS before being stained for $30 \mathrm{~min}$ at room temperature in a propidium iodide (PI)-staining solution (PBS with $20 \mu \mathrm{g} / \mathrm{ml}$ PI (Sigma), $60 \mu \mathrm{g} / \mathrm{ml}$ RNase A (Sigma) and $0.1 \% \mathrm{v} / \mathrm{v}$ Triton X-100 (Sigma)). Fluorescence emitted from the PI-DNA complex was analysed by flow cytometry, using a FACS Aria Flow Cytometer (BD Biosciences, San Jose, CA, USA). 


\section{Additional material}

Additional file 1: Specific MYCN 3'UTR knockdown mediated by the aMN-1658 shRNA. (A): The 3'UTR of MYCN was PCR-amplified from human genomic DNA with primers ON178

(5'AAAGCTGCGCACTAGTATCTGGACCAGGCTGTGGGTAGA3' -Spel site) and ON181 (5' GATCAAGCTTAATTTAAAGCTATTTATTIT 3' -HindIII site). PCR products were digested with Spel/HindIII and ligated into Spel/HindIIIdigested pMIR-REPORT vector (Invitrogen) to produce Luc-MYCN-3'UTR. A control Luc no-target (NT)-3'UTR plasmid was made by amplification of the $3^{\prime} U T R$ region from REIC using ON361

(5'GATCAAGCTTAATTTTAAGCTATTTATTTT'-Spel site) and ON327

(5'GATCAAGCTTCTATGGAAGATTITTAATACAGG3' - HindIII) as primers and ligated into Spel/Hindlll digested pMIR-REPORT vector using the In-

Fusion Dry-Down PCR Cloning Kit (Clontech). HEK-293 cells were seeded in 12-well plates, incubated for 48 hrs and transfected with a cocktail containing: $0.02 \mu \mathrm{g} \mathrm{pGL4.75[hRluc/CMV]}$ Vector (Promega), $0.1 \mu \mathrm{g} \mathrm{pMIR-}$ Report containing either the MYCN 3'UTR (LuC MYCN-3'UTR) or control (Luc-NT-3'UTR), and $1 \mu \mathrm{g}$ shRNA expressing plasmid (pScr/H1wt, paMN$887 / \mathrm{H} 1 \mathrm{wt}$ or paMN-1658/H1wt). Transfected cells were then incubated for 48 hrs before luciferase activities were measured as described.(B): Western blot analysis of MYCN and $\beta$-actin expression in SK-N-BE(2) transfected cells. CMV-MYCN (kind gift from Dr. Jason Shohet) expresses the MYCN cDNA lacking a 3'UTR. SK-N-BE(2) cells were transiently cotransfected with the shRNA-expressing plasmids (pScr/H1wt, paMN-887/ H1wt or paMN-1658/H1wt) and pCMV-MYCN (+) or the shRNAexpressing plasmids and pCMV-GFP (-). The aMN-1658 shRNA is not able to suppress expression of MYCN from the 3'UTR-lacking PCMV-MYCN plasmid. M = Magic Mark XP (Invitrogen).

Additional file 2: mRNA expression of neuronal markers in neuroblastoma cell lines after transduction with inducible antiMYCN shRNA expressing retroviruses. Real-time RT-PCR analysis of NFL (A) and GAP43 (B) mRNA expression in Kelly and SK-N-BE(2) cells transduced with the retrovirus RV-1658. Cells were incubated for the indicated numbers of days in the presence $(+)$ or absence $(-)$ of $1 \mu \mathrm{g} / \mathrm{ml}$ doxycyclin (dox). $3 / 3$ and $+/$ - indicate that the cells were incubated for 3 days in the presence of dox, followed by 3 days in the absence of dox. (C): Real-time RT-PCR analysis of ChAT, VAChT, TH and NPY mRNA in RV1658 transduced Kelly and SK-N-BE(2) cells grown in the absence $(-)$ or presence (+) of doxycyclin (dox) for 6 days.

Additional file 3: Cell cycle distribution of neuroblastoma cell lines after transduction with inducible anti-MYCN shRNA expressing retroviruses. Flow cytometric analysis showing the cell cycle distribution of Kelly (A) and SK-N-BE(2) (B) cells transduced with the RV-1658 and RV$\mathrm{Scr}$ retroviruses in the presence (+) or absence (-) of $1 \mu \mathrm{g} / \mathrm{ml}$ doxycyclin (dox).

Additional file 4: Oligonucleotides. Oligonucleotides used in this study.

\section{Acknowledgements}

This work was supported by grants from the Northern-Norwegian Health Authorities (gene therapy program) and Norwegian Cancer Society (Ragnvarda F. Sørvik and Håkon Starheims Foundation)

\section{Author details}

'Department of Pediatrics, University Hospital of North-Norway, 9038 Tromsø, Norway. ${ }^{2}$ Department of Pediatric Research, Institute of Clinical Medicine, University of Tromsø, 9037 Tromsø, Norway.

\section{Authors' contributions}

JRH designed and constructed the vectors, performed most of the experiments, drafted the manuscript, and critically planned and discussed all aspects of this work. BHH performed the clonogenic assays, participated in transduction studies. BHH, JB and TF helped to plan and critically discuss the study and participated in drafting the manuscript. ET performed several realtime RT-PCR experiments and cultivated the retrovirus transduced neuroblastoma cells. CL cultivated the cell cultures and participated in the experimental work involving cell cultures and luciferase measurements. CE designed and supervised the experimental work and wrote the final manuscript. All authors read and approved the final manuscript.

\section{Competing interests}

The authors declare that they have no competing interests.

Received: 5 August 2010 Accepted: 3 January 2011

Published: 3 January 2011

\section{References}

1. Maris JM, Hogarty MD, Bagatell R, Cohn SL: Neuroblastoma. Lancet 2007, 369:2106-2120

2. Bell E, Chen L, Liu T, Marshall GM, Lunec J, Tweddle DA: MYCN oncoprotein targets and their therapeutic potential. Cancer Lett 2010, 293:144-157.

3. Kitanaka C, Kato K, ljiri R, Sakurada K, Tomiyama A, Noguchi K, et al: Increased Ras expression and caspase-independent neuroblastoma cell death: possible mechanism of spontaneous neuroblastoma regression. J Natl Cancer Inst 2002, 94:358-368.

4. Pritchard J, Hickman JA: Why does stage $4 \mathrm{~s}$ neuroblastoma regress spontaneously? Lancet 1994, 344:869-870.

5. Bolande RP: The spontaneous regression of neuroblastoma. Experimental evidence for a natural host immunity. Pathol Annu 1991, 26(Pt 2):187-199.

6. Koizumi H, Hamano S, Doi M, Tatsunami S, Nakada K, Shinagawa T, et al. Increased occurrence of caspase-dependent apoptosis in unfavorable neuroblastomas. Am J Surg Pathol 2006, 30:249-257.

7. Pahlman S, Odelstad L, Larsson E, Grotte G, Nilsson K: Phenotypic changes of human neuroblastoma cells in culture induced by 12-0tetradecanoyl-phorbol-13-acetate. Int J Cancer 1981, 28:583-589.

8. Edsjo A, Holmquist L, Pahlman S: Neuroblastoma as an experimental model for neuronal differentiation and hypoxia-induced tumor cell dedifferentiation. Semin Cancer Biol 2007, 17:248-256.

9. Bell E, Premkumar R, Carr J, Lu X, Lovat PE, Kees UR, et al: The role of MYCN in the failure of MYCN amplified neuroblastoma cell lines to G1 arrest after DNA damage. Cell Cycle 2006, 5:2639-2647.

10. Haber M, Bordow SB, Gilbert J, Madafiglio J, Kavallaris M, Marshall GM, et al: Altered expression of the MYCN oncogene modulates MRP gene expression and response to cytotoxic drugs in neuroblastoma cells. Oncogene 1999, 18:2777-2782.

11. Negroni A, Scarpa S, Romeo A, Ferrari S, Modesti A, Raschella G: Decrease of proliferation rate and induction of differentiation by a MYCN antisense DNA oligomer in a human neuroblastoma cell line. Cell Growth Differ 1991, 2:511-518.

12. Tonelli R, Purgato S, Camerin C, Fronza R, Bologna F, Alboresi S, et al: Antigene peptide nucleic acid specifically inhibits MYCN expression in human neuroblastoma cells leading to cell growth inhibition and apoptosis. Mol Cancer Ther 2005, 4:779-786.

13. Wang SL, Yao HH, Qin ZH: Strategies for short hairpin RNA delivery in cancer gene therapy. Expert Opin Biol Ther 2009, 9:1357-1368.

14. Yu JY, DeRuiter SL, Turner DL: RNA interference by expression of shortinterfering RNAs and hairpin RNAs in mammalian cells. Proc Natl Acad Sci USA 2002, 99:6047-6052.

15. Lorens JB, Sousa C, Bennett MK, Molineaux SM, Payan DG: The use of retroviruses as pharmaceutical tools for target discovery and validation in the field of functional genomics. Curr Opin Biotechnol 2001, 12:613-621.

16. Henriksen JR, Lokke C, Hammero M, Geerts D, Versteeg R, Flaegstad T, et al: Comparison of RNAi efficiency mediated by tetracycline-responsive $\mathrm{H} 1$ and U6 promoter variants in mammalian cell lines. Nucleic Acids Res 2007, 35:e67.

17. Henriksen JR, Buechner J, økke C, Flægstad T, Einvik C: Inhibition of gene function in mammalian cells using short-hairpin RNA (shRNA). Methods in Molecular Biology 2011, 703:189-204.

18. Paddison PJ, Caudy AA, Bernstein E, Hannon GJ, Conklin DS: Short hairpin RNAs (shRNAs) induce sequence-specific silencing in mammalian cells. Genes Dev 2002, 16:948-958.

19. Breit S, Schwab M: Suppression of MYC by high expression of NMYC in human neuroblastoma cells. J Neurosci Res 1989, 24:21-28.

20. Westermann F, Muth D, Benner A, Bauer T, Henrich KO, Oberthuer A, et al: Distinct transcriptional MYCN/c-MYC activities are associated with spontaneous regression or malignant progression in neuroblastomas. Genome Biol 2008, 9:R150. 
21. Schrama $L H$, Lepperdinger $G$, Moritz $A$, van den Engel NK, Marquart $A$, Oestreicher AB, et al: B-50/growth-associated protein-43, a marker of neural development in Xenopus laevis. Neuroscience 1997, 76:635-652.

22. Yu UY, Cha JE, Ju SY, Cho KA, Yoo ES, Ryu KH, et al: Effect on Cell Cycle Progression by N-Myc Knockdown in SK-N-BE(2) Neuroblastoma Cell Line and Cytotoxicity with STI-571 Compound. Cancer Res Treat 2008, 40:27-32.

23. Bell E, Lunec J, Tweddle DA: Cell cycle regulation targets of MYCN identified by gene expression microarrays. Cell Cycle 2007, 6:1249-1256.

24. Ross RA, Biedler JL, Spengler BA, Reis DJ: Neurotransmitter-synthesizing enzymes in 14 human neuroblastoma cell lines. Cell Mol Neurobiol 1981, 1:301-311.

25. Edsjo A, Lavenius E, Nilsson H, Hoehner JC, Simonsson P, Culp LA, et al: Expression of trkB in human neuroblastoma in relation to MYCN expression and retinoic acid treatment. Lab Invest 2003, 83:813-823.

26. Bourdeaut F, Janoueix-Lerosey I, Lucchesi C, Paris R, Ribeiro A, de Pontual L, et al: Cholinergic switch associated with morphological differentiation in neuroblastoma. J Pathol 2009, 219:463-472.

27. De Preter K, Pattyn F, Berx G, Strumane K, Menten B, Van Roy F, et al: Combined subtractive CDNA cloning and array CGH: an efficient approach for identification of overexpressed genes in DNA amplicons. BMC Genomics 2004, 5:11.

28. Edsjo A, Nilsson H, Vandesompele J, Karlsson J, Pattyn F, Culp LA, et al: Neuroblastoma cells with overexpressed MYCN retain their capacity to undergo neuronal differentiation. Lab Invest 2004, 84:406-417.

29. Swift S, Lorens J, Achacoso P, Nolan GP: Rapid production of retroviruses for efficient gene delivery to mammalian cells using $293 \mathrm{~T}$ cell-based systems. Curr Protoc Immunol 2001, Chapter 10, Unit 10.17C.

30. Evensen L, Micklem DR, Blois A, Berge SV, Aarsaether N, Littlewood-Evans A, et al: Mural cell associated VEGF is required for organotypic vessel formation. PLoS One 2009, 4:e5798.

31. Hellemans J, Mortier G, De PA, Speleman F, Vandesompele J: qBase relative quantification framework and software for management and automated analysis of real-time quantitative PCR data. Genome Biol 2007, 8:R19.

doi:10.1186/1471-213X-11-1

Cite this article as: Henriksen et al: Conditional expression of retrovirally delivered anti-MYCN shRNA as an in vitro model system to study neuronal differentiation in MYCN-amplified neuroblastoma. BMC

Developmental Biology 2011 11:1.

\section{Submit your next manuscript to BioMed Central and take full advantage of:}

- Convenient online submission

- Thorough peer review

- No space constraints or color figure charges

- Immediate publication on acceptance

- Inclusion in PubMed, CAS, Scopus and Google Scholar

- Research which is freely available for redistribution

Submit your manuscript at www.biomedcentral.com/submit 